\title{
Fitness maximization by dispersal: evidence from an invasion experiment
}

\author{
D. Bonte, ${ }^{1,4}$ A. De Roissart, ${ }^{1}$ N. Wybouw, ${ }^{2}$ And T. Van Leeuwen ${ }^{2,3}$ \\ ${ }^{1}$ Ghent University, Department of Biology, Terrestrial Ecology Unit, K. L. Ledeganckstraat 35, B-9000 Ghent, Belgium \\ ${ }^{2}$ Ghent University, Department of Crop Protection, Laboratory of Agrozoology, Coupure Links 653, B-9000 Ghent, Belgium \\ ${ }^{3}$ University of Amsterdam, Institute for Biodiversity and Ecosystem Dynamics, Amsterdam, The Netherlands
}

\begin{abstract}
Dispersal is essential for population persistence in transient environments. While costs of dispersal are ubiquitous, individual advantages of dispersal remain poorly understood. Not all individuals from a population disperse, and individual heterogeneity in costs and benefits of dispersal underlie phenotype-dependent dispersal strategies. Dispersing phenotypes are always expected to maximize their fitness by adaptive decision making relative to the alternative strategy of remaining philopatric. While this first principle is well acknowledged in theoretical ecology, empirical verification is extremely difficult, due to a plethora of experimental constraints. We studied fitness prospects of dispersal in a game theoretical context using the two-spotted spider mite Tetranychus urticae as a model species. We demonstrate that dispersing phenotypes represent those individuals able to maximize their fitness in a novel, less populated environment reached after dispersal. In contrast to philopatric phenotypes, successful dispersers performed better in a low density post-dispersal context, but worse in a high density philopatric context. They increased fitness about $450 \%$ relative to the strategy of remaining philopatric. The optimization of phenotype-dependent dispersal, thus, maximizes fitness.
\end{abstract}

Key words: dispersal; intraspecific competition; invasion; philopatric phenotype; population density; Tetranychus urticae; translocation; two-spotted spider mite.

\section{INTRODUCTION}

Any active or passive attempt to move from a site of birth to another breeding site is referred to as dispersal (Clobert et al. 2009) and recognized as a key life history trait to minimize kin competition, crowding, and any consequence of deteriorating habitat quality (Bowler and Benton 2005). As such, it allows organisms to survive increasing rates of habitat fragmentation and climate change (Kokko and Lopez-Sepulcre 2006, Berg et al. 2010), but selective advantages to dispersing individuals remain poorly understood (Ronce 2007). Understanding heterogeneity in dispersal is currently crucial given the consideration of new conservation strategies (Thomas 2011a,b) that aim to translocate individuals from natal ranges into potential suitable habitat that cannot (anymore) be reached by natural dispersal. These strategies are likely to fail if the transferred individuals are not optimally adapted to the new context (Travis et al. 2013).

Advantages of dispersal generally comprise a reduction of competition with kin or non-kin by settling in low density environments (Bitume et al. 2013). There is currently a strong consensus that dispersal encompasses

Manuscript received 11 December 2013; revised 18 April 2014; accepted 29 April 2014. Corresponding Editor: T. E. X. Miller.

${ }^{4}$ E-mail: dries.bonte@ugent.be a three-stage process, including the effective departure, transfer, and settlement (Bowler and Benton 2005). Dispersal decisions at each of these stages are taken in a conditional way, i.e., according to costs levied during each of these stages relative to the benefits of the entire dispersal strategy (Clobert et al. 2009). Typical costs associated with the transfer phase are energetic or risk costs directly affecting energy reserves or survival, but costs can be equally levied during settlement. Examples include the loss of advantages from being locally adapted, the loss of prior residence advantages like a familiar environment, or losing group-living benefits (Bonte et al. 2012).

Typically, individuals from the same population vary in their morphological, physiological, or behavioral state due to both genetic and environmental factors. The phenotype can be fixed when linked to the genotype, as, for instance, is the case for personalities, or plastic due to developmental contexts generating variation in body condition (Bonte and de la Peña 2009) or settling behavior (Bonte et al. 2011). In the Glanville fritillary butterfly, dispersing phenotypes are associated with a certain phosphoglucose isomerase (pgi) genotype and have a higher metabolic rate and a larger clutch size. However, these individuals experience trade-offs with longevity under stressful conditions, while more philopatric individuals live longer. As a result, the advantages of having a dispersal phenotype may only hold under 
non-stressful conditions, for instance, when population densities are low (Bonte and Saastamoinen 2012).

Independent of the mechanisms generating phenotypic variation, philopatric and dispersing phenotypes are expected to maximize fitness under the local environmental conditions that they experience (Clobert et al. 2009). Philopatric phenotypes do not invest in dispersal because their fitness benefits are expected to be marginal relative to the costs, while benefits are expected to be much higher in those individual phenotypes that leave their natal environment. This might be especially true for competitive subordinate (Bonte et al. 2011) or asocial individuals (Cote et al. 2010a, b), rendering dispersal risk-taking an advantageous strategy. Because of dispersal costs and because individuals differ phenotypically, the fitness of dispersing individuals is not expected to be identical to those being philopatric, but always higher relative to the alternative strategy of staying home (Forero et al. 2002, Ronce 2007).

Earlier comparative studies demonstrated fitness correlates of dispersal, often interpreted as costs, but none have been able to demonstrate the principle of fitness maximization by dispersal (Bonte et al. 2012). While well established in dispersal theory (Ronce 2007), its empirical verification is extremely difficult. First, measures of fitness should be inclusive and also take into account offspring performance, because changes in population structure may have a fitness impact over several generations (Delgado et al. 2011). Second, differences in lifetime reproductive success (LRS) between philopatric and dispersive individuals may be due to physiological constraints, rather than fitness costs, for instance, when only individuals in the best condition are able to disperse long distances, or when those in subordinate condition are forced to leave (Bonte et al. 2012). As such, differences in LRS between philopatric and dispersive phenotypes do not necessarily represent dispersal costs, but may reflect phenotypedependent dispersal (Belichon et al. 1996). Third, dispersal is known to have a heritable component leading to individual consistency and parent-offspring resemblance, which raises statistical problems due to non-independency of data (Doligez and Part 2008). Finally, researchers may fail to detect (long distance) dispersing phenotypes in open populations (Belichon et al. 1996). Experimental approaches might overcome this problem by constraining emigration or translocating philopatric individuals (Johnson and Gaines 1987, Hahne et al. 2011), and should focus on understanding the state-dependency of dispersal to separate fitness differences due to body condition from dispersal costs. Dispersing individuals may, for instance, perform equally as philopatric ones overall, but, nevertheless, attain higher fitness than had they remained philopatric.

One promising avenue in evolutionary research is using mutants or transgenics to detect mechanisms driving fitness consequences of competing evolutionary strategies (Kawecki et al. 2012). When such mutants are easily detectable and do not differ in life history relative to the wild strain, they provide an excellent system to monitor the invasion success of different behavioral phenotypes in a novel context. We used a pesticidesensitive strain of the two-spotted spider mite Tetranychus urticae, of which a mutant has been selected that exhibits strong maternally inherited pesticide resistance (Van Leeuwen et al. 2006). This strong resistant phenotype was shown to be caused by mutations in cytochrome b, a mitochondrially encoded protein in the respiratory pathway (Van Leeuwen et al. 2008). Despite the unusual nature of these mutations, these resistant mites do not differ in life history traits relative to those from the baseline strain from which they have been selected (Van Leeuwen et al. 2008). In Tetranychus urticae, density and relatedness act as proximate drivers of dispersal distance (Bitume et al. 2013), and dispersal distance heritability is strongly influenced by the prevailing densities (Bitume et al. 2011). The distribution of individuals after dispersal is typically right, skewed with more individuals settling at smaller distances, and deviates significantly from a homogenous distribution, i.e., the ideal free distribution (Fretwell and Lucas 1970, Fretwell 1972, Krivan et al. 2008). This suggests already the presence of substantial dispersal costs (Bonte et al. 2012). It remains, however, challenging to understand which individuals eventually decide to disperse and to incur these costs. By means of a translocation experiment, we assessed the invasion success of dispersive and philopatric resistant phenotypes in terms of the number of offspring reaching maturity in both a philopatric and dispersive context by applying a pesticide treatment. This allowed us to directly assess genotypic success, so measuring fitness within the ecological context experienced by descendants. Because philopatric and dispersive phenotypes can be differently affected by dispersal costs (Belichon et al. 1996, Clobert et al. 2009), we simultaneously frustrated dispersers by translocating them to a philopatric context they left, and enforced philopatric phenotypes into a context they would have experienced had they dispersed. As such, we were able to keep the social context of the translocated individuals similar as under non-manipulated conditions.

\section{Material And Methods}

\section{Study species}

The spider mite Tetranychus urticae, is a haplodiploid polyphagous herbivore feeding on plant cell fluids. Because of its high fecundity and short generation time, the species causes serious damage to crops and plants in general. The species is known to engage in aerial dispersal under suitable meteorological conditions when environmental conditions are strongly deteriorating ( $\mathrm{Li}$ and Margolies 1994, Clotuche et al. 2011, 2013, De Roissart et al. 2013). The species does, however, predominantly disperse by ambulatory movements, i.e., by walking from leaf to leaf. In all cases, young 
inseminated female mites are the dominant dispersing life stage. Mites typically disperse when densities and relatedness increase (Bitume et al. 2013) and settle on uninfected leaves where densities are low. As such, the population spread follows a typical diffusive pattern leaving deteriorated plants behind the moving front. Dispersive mites can incur transfer costs because they do not feed during movement on stem tissues and because they leave the protective silk environment in the natal patch, thereby increasing vulnerability to predation and dehydration. Similar costs can be expected during settlement under low densities and may induce Allee effects (Le Goff et al. 2010). The simulated dynamics in our experiment represent this natural dispersal process, although potential mortality due to dehydration and predation is avoided, since predators are absent and aerial humidity is always high in the experimental chambers.

Two strains of $T$. urticae with a common genetic background were used during the experiments. The original bifenazate-susceptible strain (LS-VL) of $T$. urticae was collected in October 2000 from roses near Ghent, Belgium, where pesticides had not been used for at least 10 years. The BR-VL bifenazate-resistant strain was selected from LS-VL by successively applying bifenazate at concentrations that killed $90 \%$ of individuals (Van Leeuwen et al. 2008). Both strains were kept in the laboratory on potted bean plants (Phaseolus vulgaris L. cv. Prelude) under controlled conditions $(16 \mathrm{~h}$ daylight: $8 \mathrm{~h}$ darkness and $25^{\circ} \mathrm{C}$ ). Van Leeuwen et al. (2008) demonstrated that bifenazate resistance is maternally inherited and highly correlated with mutations in cytochrome b. Resistant mites show no statistical differences in the basic life history traits (fecundity, longevity, time to maturity, and sex ratio) and subsequent fitness costs in the absence of pesticide (Van Leeuwen et al. 2008).

\section{Experimental setup}

Main experiment.-Fifty females of the wild susceptible type (LS-VL strain) and mutant resistant line (BRVL strain) were collected and allowed to lay eggs for 48 hours on a fresh bean leaf $(7 \times 7 \mathrm{~cm})$ to synchronize their offspring. After hatching and juvenile development, 50 one-to-two-day old mated females were chosen at random to start the dispersal procedure. These young T. urticae females are the dominant dispersers (Bitume et al. 2011). The dispersal procedure was initiated by translocating the females to a bean leaf square that subsequently served as the source population for dispersal $(1 \times 1 \mathrm{~cm}$, in order to generate sufficiently high densities that induce emigration; see Bitume et al. 2013). This leaf fragment was connected linearly to two consecutive leaves by Parafilm bridges $(8 \times 2 \mathrm{~cm})$, forming a line of three patches. The first patch is defined as the home patch; patches 2 and 3 are the patches reached after dispersal. Wet cotton beneath the leaves kept them fresh and prevented mites from escaping.
Females were allowed to disperse for 48 hours. Each dispersal trial consisted of one series of mites $(1 \times 50$ mites) from the mutant (BR-VL) strain and three series of the wild type (LS-VL) strain $(3 \times 50$ mites $)$. We replicated this experiment, consisting of four series, 30 times. After removal of the bridges, we assessed local population sizes by counting the number of adult females on each patch (leaf).

Philopatric and dispersive mites from the resistant strain were then used as experimental subjects, assessing their reproductive performance when translocated to different patch types established by the susceptible strain (Fig. 1). For example, three resistant mites were randomly chosen from those that had dispersed to patch 3. One of these mites was then added to a population of susceptible mites in each of the three patch types, each time removing a susceptible mite so as to maintain the original population size and phenotypic context. Mites were allowed to reproduce for 14 days (until hatching of the next generation) at $25^{\circ} \mathrm{C}$ and $16 \mathrm{~h}$ daylight: $8 \mathrm{~h}$ darkness. After 14 days, all discs were sprayed with 0.8 $\mathrm{mL}(1000 \mathrm{mg} / \mathrm{L}$ bifenazate) of commercially formulated bifenazate (Floramite 240 SC, Chemtura, Middlebury, Connecticut, USA), which killed all mites of the LS-VL strain. After 24 hours, the number of surviving and dead mites in each patch was counted. In order to validate the treatment efficacy, we performed a genetic screening of more than 200 randomly selected surviving and dying mites after pesticide treatment. We followed the procedure of Van Leeuwen et al. (2008). In short, we sequenced mitochondrial DNA (mtDNA) from individual mites that had been amplified and purified using the internal primers cytbWTF 5'-CGGAATAATTTTACAAATAACTCATGC and cytbWTR 5'-TGGTACAGATCGTAGAATTGCG. All surviving mites bore the expected mutation and were descendants from the introduced resistant female, and dying mites were wild type and descendants from the susceptible baseline population.

Experimental control.-Since translocation experiments might affect the behavior of animals (Heidinger et al. 2009), we followed dispersal dynamics and subsequent population growth for the two strains under identical experimental conditions but without any translocation. The ratio of population size at 14 days to the number of founding females denotes the per capita reproductive output of a particular phenotype in a particular patch type. This average fitness measure was used as a control for the (density-dependent) individual reproductive output of the resistant female introduced in a pesticide-susceptible population, and allows an additional test of fitness differences between the two strains. By applying a pesticide treatment, we validated its effectiveness in relation to different population densities.

\section{Data analysis}

The probability distribution of spider mites among the three patch types was modeled by ordered multinomial logistic regression (see Bitume et al. 2013). The 


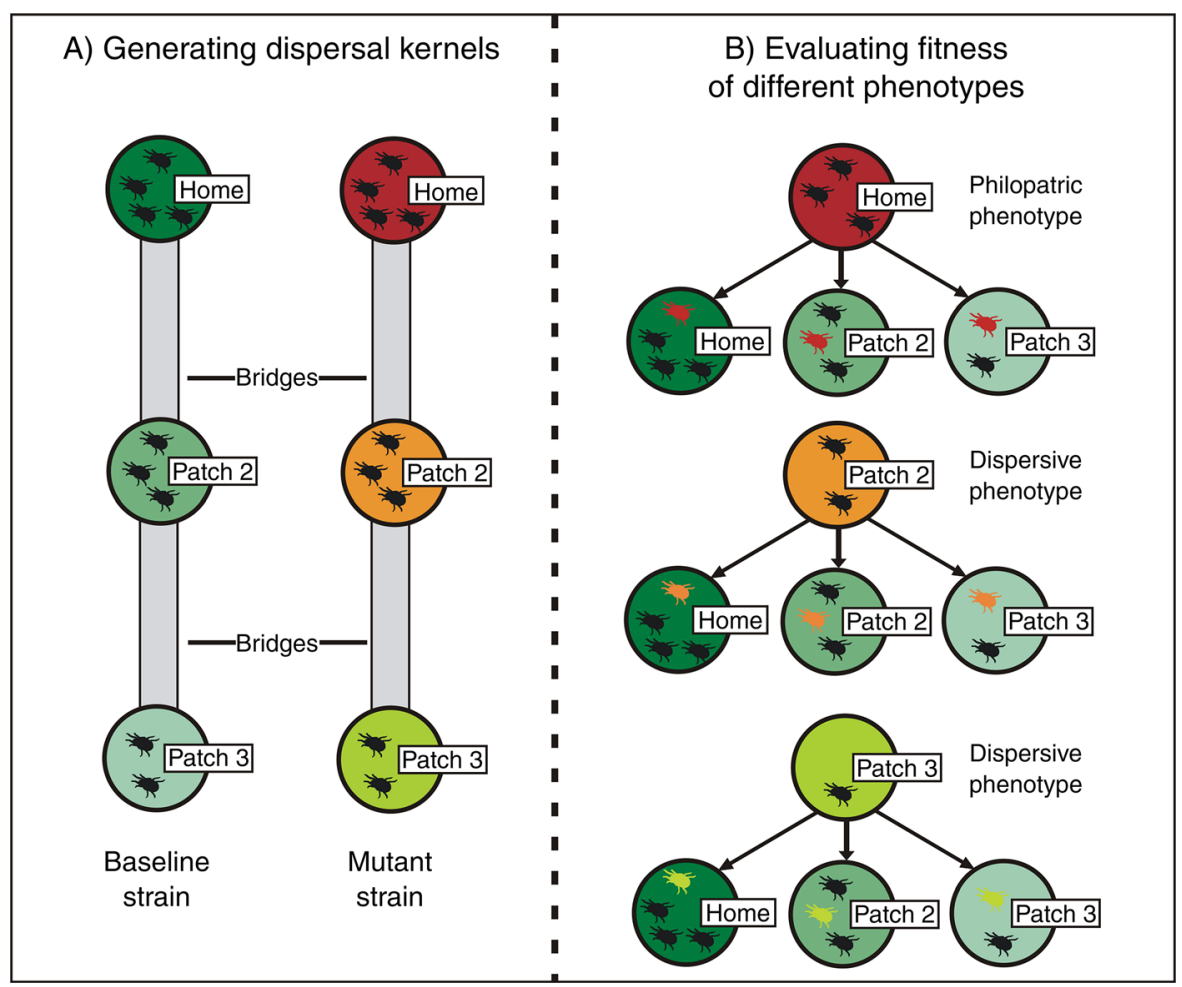

FIG. 1. Schematic representation of the experimental setup of the invasion experiment. Mites from the susceptible baseline strain and the acaracide resistant mutant strain disperse in a similar way and generate variation in density among the three connected patches (A). After 48 hours, the connecting bridges are removed and one susceptible mite from each of the three patches is replaced by a mutant resistant philopatric (from the home patch, or patch 1) or dispersive phenotype from patches 2 or 3 (B). Mutant resistant philopatric mites are represented in the color of the circle at their point of origin. By allowing reproduction, the mutant can invade the baseline population, and its invasion success as measured by the number of progeny in the next generation is assessed by applying an acaracide treatment. The experimental procedure was replicated such that 30 replicates per contextphenotype combination were obtained.

cumulative distribution of individuals across patches in the set up was modeled in relation to the strain, i.e., susceptible or resistant. We corrected for interdependency of the data within each experimental setup by including replicate as a random effect, and for potential interdependence among a set of simultaneously conducted experiments due to, for instance, common climatological conditions and similarity in host plant quality by adding day of the experimental assay as a second random component.

Generalized linear mixed models for Poisson-distributed data with log-link and correction for potential overdispersion (Verbeke and Molenberghs 2000) were used to analyze the number of descendants within the different patches after 14 days (hereafter referred to as fitness). Independent factors were the patch occupied after possible dispersal (this, then, refers to the dispersal phenotype) and the patch or density to which the female was translocated (the dispersal context). We controlled, in our analysis, for non-independence generated by performing tests at the same time periods with the same source of mites by modeling random intercepts and slopes for each replicate to correct for respectively variation in average densities and distance-effects among the used replicates. Similar analyses were performed for the per capita reproductive output relative to the patch of settlement or density in the control experiments. All analyses were done in SAS 9.2 with the GLIMMIX procedure (SAS Institute 2009). This procedure fits generalized linear mixed models by likelihood-based techniques conditional on normally distributed random effects. The GLIMMIX procedure allows data that can have any distribution in the exponential family and provides back-transformed least squares means estimates (not possible for multinomial regressions). Tukey Kramer posthoc tests were implemented to correct for multiple comparisons in the pairwise contrasts.

\section{RESUlts}

After 48 hours, on average, 21.7 individuals remained philopatric in the home patch (patch 1), 14.5 individuals dispersed to patch 2, and 3.5 individuals dispersed to patch 3 (Fig. 2). The distribution of the mites did not differ between the strains $\left(F_{1,104}=0.62 ; P=0.432\right)$, so evolved resistance did not influence the dispersal distance distribution.

When the fitness estimates were contrasted between the control experiments in which mites from the two 


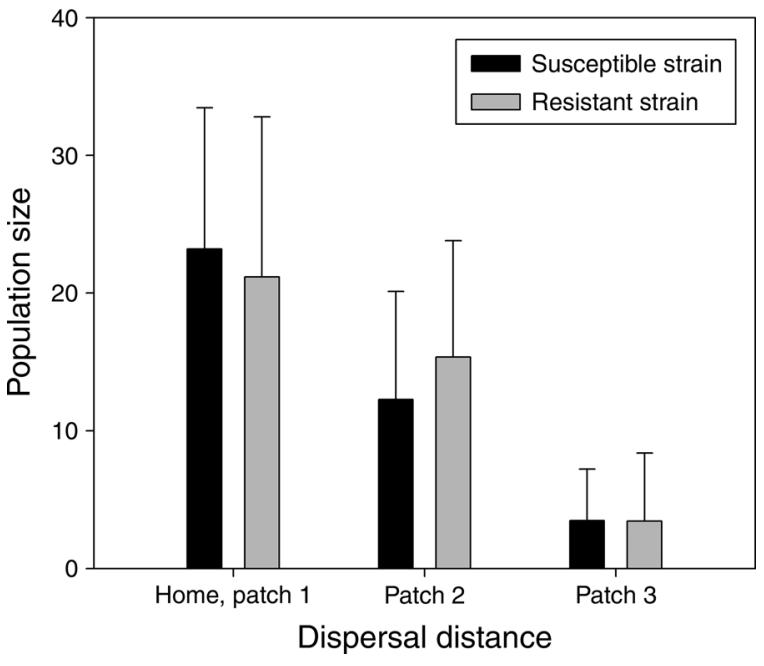

FIG. 2. Density distribution of mites from the susceptible and resistant strains after allowing dispersal. Densities declined significantly with increasing distance. Means and standard deviations are given.

strains were not translocated and the manipulated series in which a resistant individual has been introduced in its similar patch from baseline population, only patcheffects on fitness were found $\left(F_{2,57}=11.27, P<0.0001\right)$. No effects of the used strain $\left(F_{2,57}=1.02, P=0.3667\right)$ nor the interaction between the strain and the patch of dispersal were found $\left(F_{4,57}=0.43, P=0.783\right)$. So, fitness differed only according to the distance moved and was not different between the resistant and baseline strain and neither affected by the experimental manipulation. Similar effects were found in relation to density ( $\log$ [density] effect $F_{1,59}=30.20, P<0.0001$; slope $-5.0595 \pm 1.36 \mathrm{SE}$; strain effect $F_{2,59}=0.45, P=0.637$; interaction $F_{2,59}=0.43, P=0.743$ ). Density-dependent recruitment was, thus, neither different among the two strain and the implemented translocations. The pesticide treatment was $100 \%$ effective in both the susceptible and resistant strains with respectively $100 \%$ mortality and survival.

Fitness after translocation did not differ according to the distance originally dispersed by the mites (original patch $\left.F_{2,21}=0.68, P=0.519\right)$. The main effect of patch translocation was additionally significant $\left(F_{2,20}=4.61, P\right.$ $=0.020$ ), but more importantly, fitness was affected by the interaction between the patch to which a mite originally dispersed and the patch of translocation $\left(F_{4,27}\right.$ $=6.92, P=0.0006)$. This significant interaction indicates that individual phenotypes dispersing different distances showed different fitness reaction norms according to the patch to which they were transferred. Indeed, while the number of descendants remained similar for philopatric phenotypes after translocation to any patch (all $t$ values $<|1.2|, P>0.05$ after multiple comparisons), prominent fitness differences were observed for individuals that dispersed to the second or third patch, especially for those that dispersed themselves to the furthest patch (Fig. 3). The decline in fitness after translocation to a home patch was statistically significant for individuals that originally moved to the most distant patch (patch 3 phenotypes $t=-3.86$, adjusted $P=0.01$ ), and marginally significant for those that originally dispersed to the second patch $(t=-3.20$, adjusted $P=0.071)$. So, while philopatric phenotypes did not improve fitness when translocated into patches typically colonized by dispersive individuals (patches 2 and 3), dispersive phenotypes experience a statistically significant cost of $78.50 \%$ when remaining philopatric. Turned around, dispersive phenotypes increased their fitness by $458 \%$.

\section{DisCussion}

Dispersal is a complex trait consisting of distinct behavioral phases related to departure, transfer, and settlement, and will only be selected for when benefits of leaving the natal environment outweigh the costs (Clobert et al. 2009). We demonstrated that philopatric individuals reach equal fitness under different density conditions met when translocated different dispersal distances. Dispersive phenotypes, however, increased fitness by a factor of four to five by moving to new patches relative to the alternative strategy of remaining philopatric.

We, thus, demonstrate that the optimization of dispersal maximizes fitness to such an extent that substantial additional dispersal costs can be incurred. Individuals that leave the environment in which they were born do so in order to maximize their fitness in the novel environment relative to the natal environment.

While this principle of fitness maximization, being the first principle in evolutionary game theory (Nowak and Sigmund 2004), is well established in dispersal theory (Gyllenberg and Metz 2001, Poethke and Hovestadt 2002, Wild 2011), its empirical validation in nature is extremely difficult. Analyses of longitudinal data on vertebrates offer rare opportunities to measure fitness correlates of dispersal (Nevoux et al. 2013, Waser et al. 2013). Such correlative approaches, however, provide no causal evidence. Alternative experimental approaches, by constraining emigration or transferring individuals, may additionally fail to make inference of costs because such manipulations alter the population structure in both the natal and emigration environment and do not take into account multigenerational measures of fitness (Johnson and Gaines 1987, Hahne et al. 2011). We demonstrated, by a translocation experiment, using mutant mites that did not differ in life history and dispersal from their ancestor genotypes, that dispersing individuals are not a random subsample from the populations. Rather, dispersing phenotypes benefited most from released competition at distant patches and would have experienced tremendous fitness disadvantage had they remained philopatric.

The translocation experiment was set up to quantify fitness as the invasion success of one mutant that 
replaced one native wild-type individual. By applying such an approach, we kept the population (phenotypic) context of the environment similar for the focal individual as would be expected under natural conditions. However, while the mutant genotypes are selected from the susceptible base population, they can be expected to show higher levels of relatedness due to the implemented artificial selection. Such differences in relatedness may affect social interactions and eventually impact dispersal (Bitume et al. 2013) or reproductive behavior (Roeder et al. 1996, Saito 2000). Our control experiment does not indicate any difference in basic reproductive success or dispersal between the two strains, and no impact of the experimental manipulations. We are consequently confident that the observed fitness differences among the dispersal phenotypes were not due to unintended effects of the experimental manipulation.

Because different dispersal phenotypes might experience diverse dispersal costs due to, for instance, variation in morphology or physiology, care needs to be taken when interpreting the outcome of translocation experiments. Apparent absence of transfer costs might, for instance, be caused by adaptations and trade-offs in dispersive phenotypes to reduce costs of movement (Bonte et al. 2012). In our experiment, mites' fitness, on average, did not differ according to the travelled distance (no difference between individuals moving to the second or third patch). As a result, costs of transfer under natural conditions are unlikely to be related to energetic expenditure, but rather to incurred risks. While we performed experiments in an artificial environment free of potential enemies, about $20 \%$ of the adult individuals died during the transfer phase by leaving the bridges and becoming drowned. These risk costs in the experiment were of the same magnitude as benefits of dispersal in the philopatric phenotypes. If these costs are of the same order under natural conditions, they likely explain the balanced costs-benefits for dispersive individuals. Moreover, since dispersive phenotypes increased fitness in our experiments by more than $400 \%$, additional costs in more natural systems can be incurred. Under natural conditions, where mites disperse from leaf to leaf, generating patterns of diffusive spread at the population level, additional costs of moving short distances might, for instance, be induced by systemic responses, where local herbivory induces chemical defenses at other plant locations (Schmidt et al. 2009, Sarmento et al. 2011) or attract predators (Dicke et al. 1993, Van Den Boom et al. 2004, Ament et al. 2010).

While the translocation experiment allows inference of costs related to the integration into a novel context in both philopatric and dispersive phenotypes, it does not allow a full inference of the costs associated with transfer. Dispersive phenotypes cannot be forced to remain philopatric while philopatric phenotypes cannot be forced to move actively to a new location (dispersal behavior determines, after all, the phenotype). This problem is inevitable in translocation studies like this

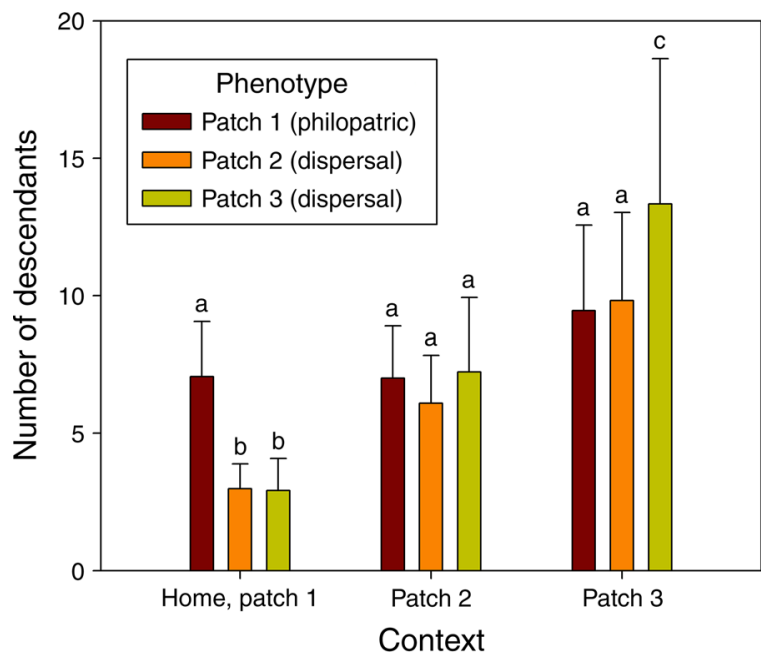

FIG. 3. Fitness measured as the number of descendants in the next generation of the translocated mites in relation to their dispersed distance (phenotype) and context of assisted immigration. Fitness of the dispersive phenotypes was maximized when transferred to a low density context reached after dispersal. Fitness of philopatric phenotypes was independent of the density context. Means and standard deviations are given. Different lowercase letters above bars indicate statistically significant differences. Except for the difference of patch 2 phenotypes among the first and second patch $(P=0.07)$, all $P<$ 0.05 after correction for multiple comparisons among all combinations.

and mainly relevant when dispersal depends on different morphs or a size (which is not the case in our study system). However, in order to estimate the magnitude of transfer costs in the dispersive phenotypes, we used a three-patch system in which potential energetic costs of displacement can be quantified.

Fitness of dispersing phenotypes in a philopatric context was about one-half that of philopatric phenotypes, while philopatric phenotypes performed equally well in all contexts. As a result, only dispersing phenotypes were negatively impacted by elevated levels of intraspecific competition met under natal conditions. Under high densities, especially subordinate individuals are expected to experience increased competitive interactions during foraging, either directly through behavioral interference or indirectly due to an increased rate of resource depletion, necessitating an increased investment into foraging movements. Such interactions lead, on average, to energy loss that cannot be invested in reproduction (Pyke et al. 1977) and to movement from high density to low density environments (Bitume et al. 2013). High densities are, therefore, expected to induce dispersal responses in those individuals that suffer most from increased intraspecific competition.

While this intraspecific competition likely drives the phenotype dependent dispersal in our experiment, it is not unlikely that the effect is strengthened by kin competition. A tight kin structure is expected under natural conditions, where populations are founded by 
few inseminated females, and, in our experiment, where individuals were collected from a lab culture. In environments where dispersal costs are high and environments are spatiotemporally stable, kin competition is the major driver of dispersal (Bonte and de la Peña 2009). Subordinate competitors can then be the dispersing phenotype, depending on the competition dynamics and metapopulation structure (Gyllenberg et al. 2008, 2011, Bonte and de la Peña 2009, Kisdi et al. 2012). These individuals, then, leave the population, thereby incurring dispersal costs in order to reduce competition. Interestingly, instead of reaching equal or lower levels of fitness relative to philopatric phenotypes transferred to a low density context, dispersive individuals benefit even more from released competition. So, inferior competitors in natal environments can be superior ones in novel environments reached after dispersal. We explicitly acknowledge that, in our study, phenotypic variation is likely not related to genotypic polymorphisms but more to variation in body condition due to differentiation in resource acquisition during development. However, the retrieved pattern strongly resembles mechanisms of personality-driven dispersal strategies with, in our case, subordinates having a lower reproductive capacity, being the more asocial phenotype (Cote et al. 2010a), thereby maximally benefiting from settlement in low density populations or groups.

Our invasion approach forces the integration of different phenotypes in either a similar or different context from which they originate. The dynamics, as simulated in our experimental setup, reflect patterns of diffusional spread as encountered during range expansions (Kubisch et al. 2013) or pest outbreaks (Kareiva 1983). It remains to be tested whether insights from this experiment can be generalized to organisms inhabiting more saturated environments where reinforced colonization is the rule. For instance, in mammals and birds (Murray 1967, Matthysen 2005), dispersal benefits are less related to the release of intraspecific competition, but merely by avoidance of kin competition, inbreeding avoidance, or risk-spreading.

In conclusion, we demonstrate that dispersing phenotypes represent those individuals able to maximize their fitness in a novel, less populated environment reached after dispersal. In contrast to philopatric phenotypes, successful dispersive Tetranychus urticae performed better in a low density post-dispersal context, but worse in a high density philopatric context. They increased fitness with about $400 \%$ relative to the strategy of remaining philopatric. We, thus, provide the first empirical evidence that the optimization of phenotypedependent dispersal maximizes individual fitness.

\section{ACKNOWLEDGMENTS}

D. Bonte, A. De Roissart, and T. Van Leeuwen designed the study; A. De Roissart and N. Wybouw performed the research; and D. Bonte and A. De Roissart wrote the first draft of the manuscript, analyzed the data, and are joint first authors of the paper. All authors were involved in the revision. D. Bonte and
T. Van Leeuwen were funded by FWO project G.0610.11. J.M.B. D. Bonte received additional support from the FWO Research Network EVENET. T. Van Leeuwen is a postdoctoral fellow of the FWO. A. De Roissart was funded by BOF project B/11668/02. N. Wybouw is an IWT doctoral fellow. We thank Hannele Penson and Ruben Mistiaen for assistance with the experiments. We are grateful for the constructive comments provided by Jean Clobert, Dustin Marshall, and Calvin Dytham.

\section{Literature Cited}

Ament, K., V. Krasikov, S. Allmann, M. Rep, F. L. W. Takken, and R. C. Schuurink. 2010. Methyl salicylate production in tomato affects biotic interactions. Plant Journal 62:124-134.

Belichon, S., J. Clobert, and M. Massot. 1996. Are there differences in fitness components between philopatric and dispersing individuals? Acta Oecologica - International Journal of Ecology 17:503-517.

Berg, M. P., E. T. Kiers, G. Driessen, M. van der Heijden, B. W. Kooi, F. Kuenen, M. Liefting, H. A. Verhoef, and J. Ellers. 2010. Adapt or disperse: understanding species persistence in a changing world. Global Change Biology 16: 587-598.

Bitume, E. V., D. Bonte, S. Magalhaes, G. S. Martin, S. Van Dongen, F. Bach, J. M. Anderson, I. Olivieri, and C. M. Nieberding. 2011. Heritability and artificial selection on ambulatory dispersal distance in Tetranychus urticae: effects of density and maternal effects. PLoS ONE 6.

Bitume, E. V., D. Bonte, O. Ronce, F. Bach, E. Flaven, I. Olivieri, and C. M. Nieberding. 2013. Density and genetic relatedness increase dispersal distance in a subsocial organism. Ecology Letters 16:430-437.

Bonte, D., and E. de la Peña. 2009. Evolution of body condition-dependent dispersal in metapopulations. Journal of Evolutionary Biology 22:1242-1251.

Bonte, D., N. De Meester, and E. Matthysen. 2011. Selective integration advantages when transience is costly: immigration behavior in an agrobiont spider. Animal Behaviour 81: 837-841.

Bonte, D., and M. Saastamoinen. 2012. Dispersal syndromes in spiders and butterflies. Pages 161-170 in J. Clobert, M. Baguette, T. Benton, and J. Bullock, editors. Dispersal and spatial evolutionary ecology. Oxford University Press, Oxford, UK.

Bonte, D., et al. 2012. Costs of dispersal. Biological Reviews 87: 290-312.

Bowler, D. E., and T. G. Benton. 2005. Causes and consequences of animal dispersal strategies: relating individual behavior to spatial dynamics. Biological Reviews 80:205225.

Clobert, J., J. F. Le Galliard, J. Cote, S. Meylan, and M. Massot. 2009. Informed dispersal, heterogeneity in animal dispersal syndromes and the dynamics of spatially structured populations. Ecology Letters 12:197-209.

Clotuche, G., A. C. Mailleux, A. A. Fernandez, J. L. Deneubourg, C. Detrain, and T. Hance. 2011. The formation of collective silk balls in the spider mite Tetranychus urticae Koch. PLoS ONE 6(4):e18854.

Clotuche, G., M. Navajas, A. C. Mailleux, and T. Hance. 2013. Reaching the ball or missing the flight? Collective dispersal in the two-spotted spider mite Tetranychus urticae. PLoS ONE 8(10):e77573.

Cote, J., J. Clobert, T. Brodin, S. Fogarty, and A. Sih. $2010 a$. Personality-dependent dispersal: characterization, ontogeny and consequences for spatially structured populations. Philosophical Transactions of the Royal Society B 365: 4065-4076.

Cote, J., S. Fogarty, K. Weinersmith, T. Brodin, and A. Sih. 2010b. Personality traits and dispersal tendency in the 
invasive mosquitofish (Gambusia affinis). Proceedings of the Royal Society B 277:1571-1579.

Delgado, M. D., I. I. Ratikainen, and H. Kokko. 2011. Inertia: the discrepancy between individual and common good in dispersal and prospecting behavior. Biological Reviews 86: 717-732.

De Roissart, A., E. de la Peña, L. Van Oyen, T. Van Leeuwen, D. J. Ballhorn, and D. Bonte. 2013. The presence of rootfeeding nematodes (not AMF) affects an herbivore dispersal strategy. Acta Oecologica-International Journal of Ecology $52: 38-44$.

Dicke, M., P. Vanbaarlen, R. Wessels, and H. Dijkman. 1993. Herbivory induces systemic production of plant volatiles that attract predators of the herbivore: extraction of endogenous elicitor. Journal of Chemical Ecology 19:581-599.

Doligez, B., and T. Part. 2008. Estimating fitness consequences of dispersal: a road to 'know-where'? Non-random dispersal and the underestimation of dispersers' fitness. Journal of Animal Ecology 77:1199-1211.

Forero, M. G., J. A. Donazar, and F. Hiraldo. 2002. Causes and fitness consequences of natal dispersal in a population of black kites. Ecology 83:858-872.

Fretwell, S. D. 1972. Populations in a seasonal environment. Princeton University Press, Princeton, New Jersey, USA.

Fretwell, S. D., and H. L. Lucas. 1970. On territorial behavior and other factors influencing habitat distribution in birds. I. Theoretical Development. Acta Biotheoretica 19:16-36.

Gyllenberg, M., E. Kisdi, and M. Utz. 2008. Evolution of condition-dependent dispersal under kin competition. Journal of Mathematical Biology 57:285-307.

Gyllenberg, M., E. Kisdi, and M. Utz. 2011. Body conditiondependent dispersal in a heterogeneous environment. Theoretical Population Biology 79:139-154.

Gyllenberg, M., and J. A. J. Metz. 2001. On fitness in structured metapopulations. Journal of Mathematical Biology 43:545560.

Hahne, J., T. Jenkins, S. Halle, and G. Heckel. 2011. Establishment success and resulting fitness consequences for vole dispersers. Oikos 120:95-105.

Heidinger, I. M. M., H. J. Poethke, D. Bonte, and S. Hein. 2009. The effect of translocation on movement behavior: a test of the assumptions of behavioral studies. Behavioural Processes 82:12-17.

Johnson, M. L., and M. S. Gaines. 1987. The selective basis for dispersal of the prairie vole, Microtus ochrogaster. Ecology 68:684-694.

Kareiva, P. M. 1983. Local movement in herbivorous insects: applying a passive diffusion model to mark-recapture field experiments. Oecologia 57:322-327.

Kawecki, T. J., R. E. Lenski, D. Ebert, B. Hollis, I. Olivieri, and M. C. Whitlock. 2012. Experimental evolution. Trends in Ecology and Evolution 27:547-560.

Kisdi, E., M. Utz, and M. Gyllenberg. 2012. Evolution of condition-dependent dispersal. Pages 139-151 in M. B. J. Clobert, T. Benton, and J. Bullock, editors. Dispersal ecology and evolution. Oxford University Press, Oxford, UK.

Kokko, H., and A. Lopez-Sepulcre. 2006. From individual dispersal to species ranges: perspectives for a changing world. Science 313:789-791.

Krivan, V., R. Cressman, and C. Schneider. 2008. The ideal free distribution: a review and synthesis of the game-theoretic perspective. Theoretical Population Biology 73:403-425.

Kubisch, A., R. D. Holt, H.-J. Poethke, and E. A. Fronhofer. 2013. Where am I and why? Synthesizing range biology and the eco-evolutionary dynamics of dispersal. Oikos 123(1):522

Le Goff, G. J., A. C. Mailleux, C. Detrain, J. L. Deneubourg, G. Clotuche, and T. Hance. 2010. Group effect on fertility, survival, and silk production in the web spinner Tetranychus urticae (Acari: Tetranychidae) during colony foundation. Behaviour 147:1169-1184.

Li, J. B., and D. C. Margolies. 1994. Responses to direct and indirect selection on aerial dispersal behavior in Tetranychus urticae. Heredity 72:10-22.

Matthysen, E. 2005. Density-dependent dispersal in birds and mammals. Ecography 28:403-416.

Murray, B. G. 1967. Dispersal in vertebrates. Ecology 48:975978.

Nevoux, M., D. Arlt, M. Nicoll, C. Jones, and K. Norris. 2013. The short- and long-term fitness consequences of natal dispersal in a wild bird population. Ecology Letters 16:438445.

Nowak, M. A., and K. Sigmund. 2004. Evolutionary dynamics of biological games. Science 303:793-799.

Poethke, H. J., and T. Hovestadt. 2002. Evolution of density- and patch-size-dependent dispersal rates. Proceedings of the Royal Society B 269:637-645.

Pyke, G. H., H. R. Pulliam, and E. L. Charnov. 1977. Optimal foraging: a selective review of theory and tests. The Quarterly Review of Biology 52:137-154.

Roeder, C., R. Harmsen, and S. Mouldey. 1996. The effects of relatedness on progeny sex ratio in spider mites. Journal of Evolutionary Biology 9:143-151.

Ronce, O. 2007. How does it feel to be like a rolling stone? Ten questions about dispersal evolution. Annual Review of Ecology, Evolution, and Systematics 38:231-253.

Saito, Y. 2000. Do kin selection and intra-sexual selection operate in spider mites? Experimental and Applied Acarology 24:351-363.

Sarmento, R. A., F. Lemos, P. M. Bleeker, R. C. Schuurink, A. Pallini, M. G. Almeida Oliveira, E. R. Lima, M. Kant, M. W. Sabelis, and A. Janssen. 2011. A herbivore that manipulates plant defense. Ecology Letters 14:229-236.

SAS Institute. 2009. SAS version 9.2. SAS Institute, Cary, North Carolina, USA.

Schmidt, L., U. Schurr, and U. S. R. Roese. 2009. Local and systemic effects of two herbivores with different feeding mechanisms on primary metabolism of cotton leaves. Plant Cell and Environment 32:893-903.

Thomas, C. D. 2011a. Anthropocene park? No alternative. Trends in Ecology and Evolution 26:497-498.

Thomas, C. D. $2011 b$. Translocation of species, climate change, and the end of trying to recreate past ecological communities. Trends in Ecology and Evolution 26:216-221.

Travis, J. M. J., et al. 2013. Dispersal and species responses to climate change. Oikos 11:1532-1540.

Van Den Boom, C. E. M., T. A. Van Beek, M. A. Posthumus, A. De Groot, and M. Dicke. 2004. Qualitative and quantitative variation among volatile profiles induced by Tetranychus urticae feeding on plants from various families. Journal of Chemical Ecology 30:69-89.

Van Leeuwen, T., L. Tirry, and R. Nauen. 2006. Complete maternal inheritance of bifenazate resistance in Tetranychus urticae Koch (Acari: Tetranychidae) and its implications in mode of action considerations. Insect Biochemistry and Molecular Biology 36:869-877.

Van Leeuwen, T., B. Vanholme, S. Van Pottelberge, P. Van Nieuwenhuyse, R. Nauen, L. Tirry, and I. Denholm. 2008. Mitochondrial heteroplasmy and the evolution of insecticide resistance: non-mendelian inheritance in action. Proceedings of the National Academy of Sciences USA 105:5980-5985.

Verbeke, G., and G. Molenberghs. 2000. Linear mixed models for longitudinal data. Springer Series in Statistics. SpringerVerlag, New York, New York, USA.

Waser, P. M., K. M. Nichols, and J. D. Hadfield. 2013. Fitness consequences of dispersal: is leaving home the best of a bad lot? Ecology 94:1287-1295.

Wild, G. 2011. Direct fitness for dynamic kin selection. Journal of Evolutionary Biology 24:1598-1610. 